\title{
APORTES Y RELEVANCIA DE COMISIONES NACIONALES EN MACROECONOMÍA Y SALUD PARA PAÍSES DE AMÉRICA LATINA
}

\author{
Armando Arredondo López*
}

\begin{abstract}
Arredondo-López A. Aportes y relevancia de comisiones nacionales en macroeconomía y salud para países de américa latina. Hitos de Ciencias Económico Administrativas 2013;19 (53):39-46
\end{abstract}

\section{RESUMEN}

El presente artículo incluye los principales planteamientos sobre las experiencias y balances de las Comisiones Nacionales en Macroeconomía y Salud (CMES), centrando su análisis en la pertinencia y relevancia para los países de América Latina. A manera de introducción, la primera parte plantea algunas premisas de las relaciones entre reforma en salud, inversión y desarrollo, como parte del eje central que abordan las CMES en los países. Posteriormente, se dan los principales antecedentes, lineamientos, conformación e implementación de tales comisiones en el mundo y en países de América Latina. La segunda parte del documento incluye el análisis de aportes y avances de metas y estrategias como objeto de análisis de tales comisiones: indicadores de mortalidad, indicadores de financiamiento/inversión e indicadores de generación de conocimiento. Finalmente, a manera de conclusión se plantean de manera explícita las principales reflexiones a partir del desarrollo de las CMES y de las tendencias de los indicadores revisados, así como una lista de sugerencias a manera de lecciones aprendidas que podrían ser retomadas para el redimensionamiento de las comisiones ya implementadas; o bien, para la implementación de nuevas comisiones en países donde aún se encuentran de manera incipiente. Contributions and relevance of National Commissions on Macroeconomics and Health for Latin American countries. Hitos de Ciencias Económico Administrativas 2013;19 (53):39-46.

\section{ABSTRACT}

This article covers the main approaches based on the experience and reports of the National Commissions on Macroeconomics and Health (CMES), focusing their analysis on the relevance and significance for the Latin American countries. As an introduction, the first part of this paper poses some premises of the relationship between health reform, investment, and development as part of the central axis that the CMES approach in the countries. After that, the main background, guidelines, creation, and implementation of such commissions in the world and in LatinAmerica are given. The second part of this document includes an analysis of contributions and goals and strategies advancement as an object of analysis of such committees: mortality, finance/ investment, and knowledge generation indicators. Finally, as a conclusion, there is a detailed explanation of the main reflections from the development of the CMES and trends of the revised indicators, as well as a list of suggestions that can be considered as learned lessons that could be retaken up for the remodeling of the already implemented commissions or to the implementation of new committees in countries that have them still in a fledgling way.
Palabras clave: Comisiones de macroeconomía y salud. Aportes. Indicadores.
Key words: Macroeconomics y health committees. Contributions. Indicators.

DIRECCIÓN PARA RECIBIR CORRESPONDENCIA: Correo electrónico: armando.arredondo@insp.mx

* Investigador Titular del Instituto Nacional de Salud Pública -México.

Fecha de recibido: 9 de diciembre de 2012 Fecha de aceptación: 18 enero de 2013. 
I desarrollo e implementación de las CMES en los países constituye una estrategia de apoyo en los procesos de reforma de la salud a nivel mundial. Ciertamente, las CMES proponen como eje temático diversas líneas de acción para el monitoreo y seguimiento de estrategias de reforma para el avance de los vínculos que existen entre economía, inversión, salud y desarrollo que se han demostrado en diversos estudios (1-3).

En efecto, estudios sobre gasto catastrófico en salud por parte de las familias (4-7), así como estudios referentes a efectos de las reformas en salud, variables macroeconómicas y equidad (8-11), ponen de manifiesto la alta prioridad y pertinencia en el desarrollo y seguimiento de variables macroeconómicas por su estrecha relación con los indicadores de salud a partir de la implementatción de las reformas.

A partir del año 2000 durante la cumbre mundial de Naciones Unidas y paralelo al acuerdo de las metas del milenio, se propuso la Comisión sobre Macroeconomía y Salud, con la idea de implementar metas y estrategias para monitorear, evaluar y retomar las evidencias empíricas sobre el establecimiento de líneas de acción que permitieran avanzar en materia de reformas de los sistemas de salud (12-14). Tal seguimiento y monitoreo se centra y coincide de manera importante con algunos de los "Objetivos del Milenio», sobre todo con los asociados a los problemas de equidad, desigualdad y rezago en materia de inversión en salud para contrarrestar los efectos negativos de mortalidad materno-infantil y problemas de salud pública como Malaria, Tuberculosis y SIDA, principalmente en países de ingreso medio y bajo.

En respuesta a dicha problemática, durante la cumbre del 2000 de ONU, los países acordaron, nuevas estrategias para ampliar el acceso de grupos prioritarios a servicios de salud. Tales estrategias dieron origen a la Comisión de Macroeconomía y Salud (CMES), planteando que se requiere de monitoreo y seguimiento de reformas organizativas de los Sistemas de Salud, que garanticen mayor inversión y cobertura universal en intervenciones prioritarias para cada país (15). Para tal efecto, agencias internacionales (OMS, BM, ONU, BID), conformaron un comité directivo de países donantes (ingreso alto) y países receptores (ingreso medio-bajo). Este comité asumió desarrollar, coordinar y supervisar estrategias de movilización de recursos para avanzar en materia de salud, economía y desarrollo. El principal acuerdo multilateral que surge de tales estrategias es que se establecen dos nuevos mecanismos de financiación e inversión en salud para darle sustento y factibilidad a las CMES en cada país (16).

En este contexto, la implementación de las CMES en cada uno de los países pasó por 3 fases fundamentales. La primer fase se refirió al desarrollo de estrategias para generar un alto nivel de toma de conciencia y apoyo mediante una divulgación amplia de los conceptos de macroeconomía y salud; Para la segunda fase de implementación, los países llevaron a cabo una evaluación integral de su situación de salud (requerimientos en matería de servicios, investigación y formación de recursos) y un análisis de su infraestructura en el área de sistemas de salud; finalmente la tercera fase, se inició en cada país con la implementación del plan de inversión en salud y el desarrollo de un mecanismo destinado a supervisar el proceso y el impacto a mediano y largo plazo en indicadores específicos de salud (17).

Una vez cumplidas las 3 fases señaladas, más de 40 países de ingreso bajo y medio de todo el mundo deciden implementar elementos del enfoque de macroeconomía y salud. De estos 40 países, solo 30 lograron implementar de manera formal las CMES bajo el liderazgo de los sectores de salud y desarrollo social. En tales países las comisiones participan de manera proactiva en el monitoreo e implementación de planes nacionales de salud en alcance a las metas del Milenio-ONU. Incluso en 15 países del Caribe inglés y 6 de América Latina, las CMES participaron de manera relevante en la ampliación de intervenciones en relación al costo-efectividad, dependiendo de la fase de reforma $y$ de los niveles de gobernanza y equidad con que operan los sistemas de salud en cada país (18-20). En este contexto, el presente artículo pretende mostrar 
algunas evidencias de aportes y lecciones aprendidas de la implementación de las CMES en el monitoreo y seguimiento de indicadores de salud planteados por los Objetivos del Milenio en relación estrecha a dichas comisiones.

\section{Consideraciones metodológicas}

Con el objeto de realizar un análisis y revisión de las tendencias cualitativas y cuantitativas de los principales indicadores que están bajo el monitoreo y seguimiento de las CMES se desarrollaron los siguientes procedimientos metodológicos:

\section{- REVISIÓN Y ANÁLSIS DE DOCUMENTOS}

CLAVE 2000- 2010. Fueron seleccionados informes, publicaciones e indicadores básicos relacionados al análisis de macroeconomía y salud para el período 2000-2010, publicados por organismos internacionales. Tales informes incluyeron el reporte mundial de la comisión, informes nacionales y regionales, particularmente para América Latina y el Caribe.

\section{- SELECCIÓN DE PAISES}

Para realizar el análisis comparativo y revisión de tendencias de indicadores cualitativos y cuantitativos, fueron seleccionados 8 países. Para ello se consideraron los siguientes criterios de selección: nivel de ingreso per cápita, ubicación geográfica en región de América Latina, fase de implementación sobre la CMES y diferentes fases de reforma en salud. Los países seleccionados fueron: México, Colombia, Brasil, Argentina, Bahamas, Haití, El Salvador y Cuba. Para fines de comparación con países de ingreso alto de la región se incluyó también EUA y Canadá.

\section{- INTEGRACIÓN DE INDICADORES}

La integración de indicadores de niveles y tendencias epidemiológicas se realizó a través de consulta electrónica en bases de datos y hojas de reporte de la base de datos del informe 2010 de las metas del Milenio-ONU-2010. Específicamente se consultaron las hojas de excel de las bases de datos del Informe 2010 de los «Objetivos del
Milenio» referidas a mortalidad infantil, mortalidad materna, tendencias de prevalencia y tratamiento referidas a tuberculosis, malaria y SIDA. Los indicadores de gasto e inversión en salud se obtuvieron a partir del informe 2009 de indicadores básicos para la región de las Américas de OPS-OMS (21).

\section{RESULTADOS}

Los resultados se presentan en dos secciones. La primer sección, aborda el análisis de indicadores sobre las metas de los «Objetivos del Milenio» que están bajo el monitoreo de las CMES, principalmente los referentes a mortalidad infantil, mortalidad materna, gasto e inversión en salud, tendencias en atención a enfermedades infecciosas (Tuberculosis, Malaria y SIDA). La segunda sección hace un análisis comparando las tendencias con las metas alcanzadas para algunos indicadores seleccionados y el grado de avance en función de las metas programadas al 2015 para cada país.

\section{Resultados en niveles y tendencias de indicadores} Las tendencias en la mortalidad infantil en menores de 5 años han tenido un comportamiento regular con tendencias decrecientes en todos los países

TABLA I. TENDENCIAS EN TASA DE MORTALIDAD INFANTIL PARA PAISES SELECCIONADOS (MENORES DE 5 AÑOS POR 1000 NACIDOS VIVOS)

\begin{tabular}{lccccc}
\hline País & 1990 & 1995 & 2000 & 2005 & 2008 \\
\hline México & 45 & 37 & 26 & 20 & 17 \\
Brasil & 56 & 36 & 28 & 22 & 18 \\
Argentina & 29 & 24 & 21 & 17 & 16 \\
Bahamas & 25 & 23 & 20 & 16 & 13 \\
Haití & 151 & 141 & 109 & 84 & 72 \\
Cuba & 14 & 11 & 9 & 7 & 6 \\
Colombia & 35 & 31 & 26 & 22 & 20 \\
El salvador & 62 & 49 & 33 & 23 & 18 \\
EUA & 11 & 9 & 8 & 8 & 8 \\
Canadá & 8 & 7 & 7 & 6 & 6 \\
\hline
\end{tabular}

Fuente: Elaboración propia a partir de Metas del Milenio. Informe 2010, ONU. 
seleccionados para este análisis. Los casos que mas llaman la atención por su nivel de mejora en los indicadores son Brasil y El Salvador pasando de 1990 al 2008 , tasas de 56 a 18 y de 62 a 18 , respectivamente. Otro caso que llama la atención por sus altas tasas de mortalidad es el caso de Haití, a pesar de que tiene tendencias decrecientes se mantiene con una tasa relativamente alta para la media de América Latina, (ver tabla I).

Respecto a las tendencias en la lucha contra el $\mathrm{VIH}$, comparando los niveles de 2001 vs. 2008, Ilaman la atención los altos niveles de mortalidad en México, Brasil, Haití y Colombia. Sobre todo las tendencias incrementales en los casos de México, Brasil, Argentina y EISalvador. También llama particularmente la atención que sólo en el caso de Colombia existen tendencias decrecientes en la mortalidad por $\mathrm{VIH}$, (ver figura 1 ).

En lo referente al número de nuevos casos de población mayor de 15 años con $\mathrm{VIH}$, los 2 países

FIGURA 1. TASAS DE MORTALIDAD Y PREVALENCIA POR VIH (POBLACIÓN MAYOR DE 15 AÑOS CON 2001 VS 2008)

\section{MORTALIDAD}

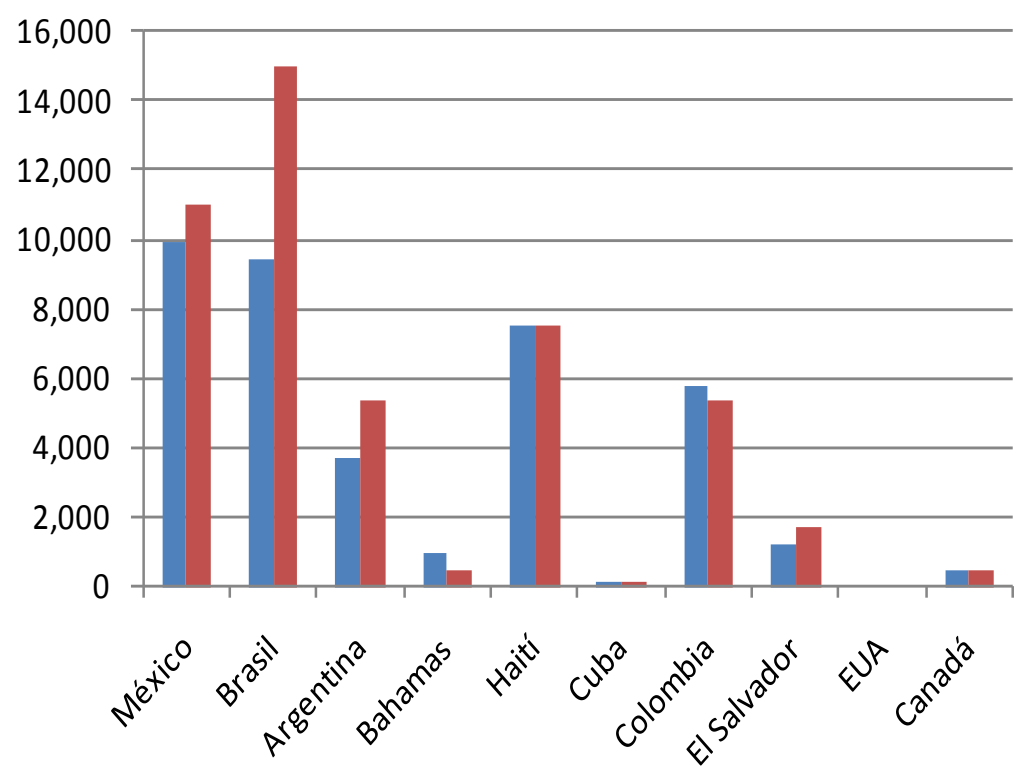

PREVALENCIA

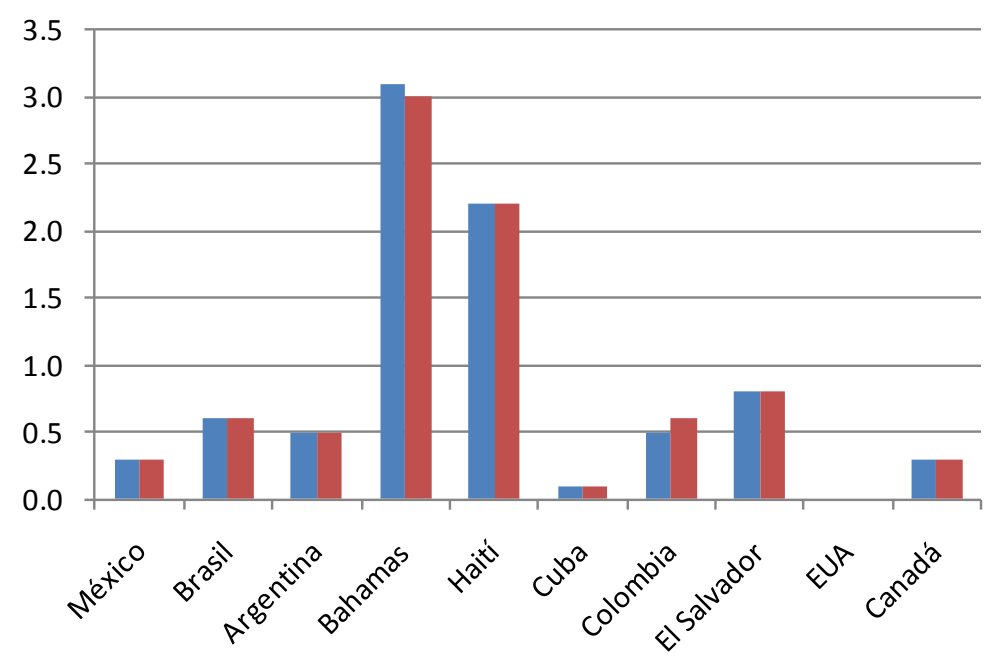

2001

2008 que más llaman la atención por sus altos niveles para el período 2001-2008, son Bahamas y Haití. Otro país que llama la atención no por sus altos niveles sino por su tendencia creciente para el período es Colombia, de hecho este país es el único que muestra tendencias crecientes constantes, (ver figura 1).

En lo referente al combate para controlar la prevalencia de tuberculosis por 100000 habitantes, en todos los países bajo análisis, los niveles y tendencias guardan un comportamiento regular, decreciente y constante para el período 1990-2008; Sin embargo en el caso de Haití, se observa una tendencia irregular, con altos niveles poblacionales y con una tasa creciente muy alta para el año 2000, y aunque tiende a decrecer permanece alta en comparación con el resto de los países, ( ver figura 2).

Sobre los niveles y tendencias de casos de paludismo tienen un comportamiento diferencial muy marcado en los países.

Fuente: Elaboración propia a partir de Metas del Milenio. Informe 2010, ONU. 
FIGURA 2. TENDENCIAS EN LA TASA DE PREVALENCIA DE TB POR 1000000 HABITANTES: 1990,2000 Y 2008

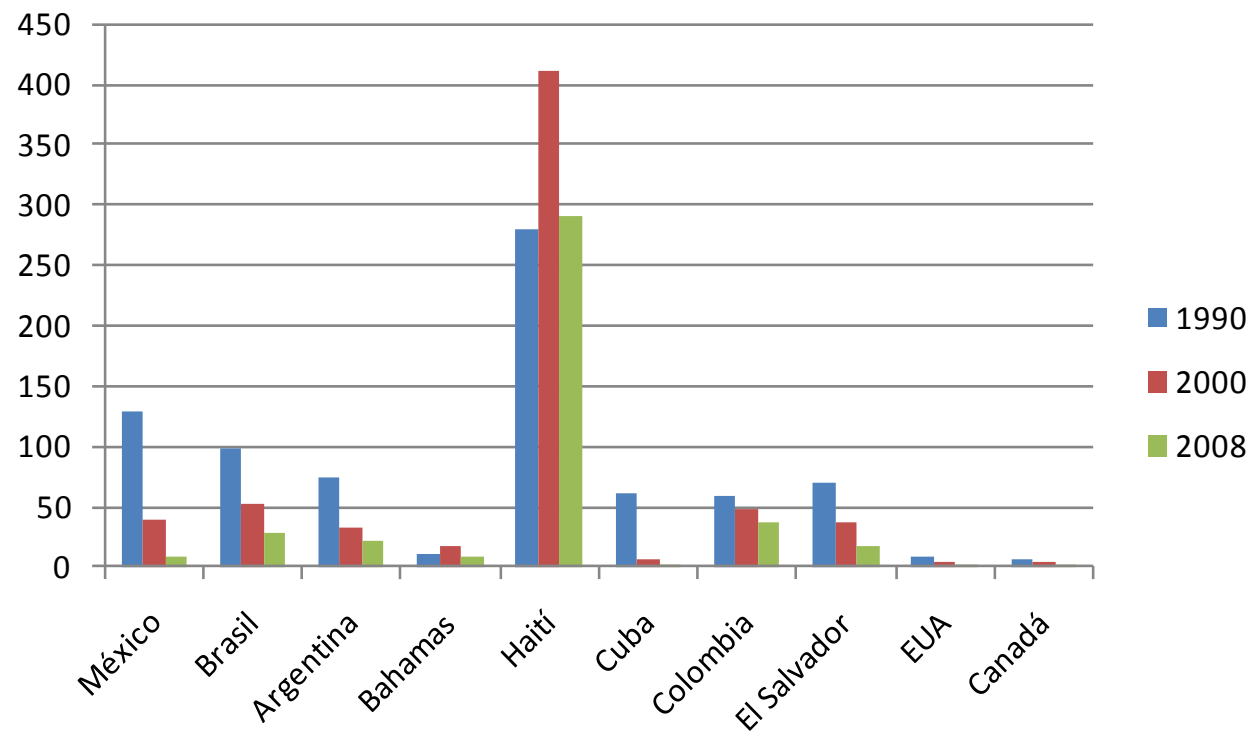

Fuente: Elaboración propia a partir de Metas del Milenio. Informe 2010, ONU.
Haití seguramente hay una amplia asociación con los niveles y condiciones de vida de este país, mientras que en los caos de Brasil y Colombia seguramente son casos altamente relacionados con comunidades marginadas en los alrededores del amazonas.

Respecto al análisis de tendencias del gasto en salud, como puede verse en la Tabla II, las tendencias del gasto en salud para el período 2002-2007, presenta tendencias crecientes
Llama la atención el alto nivel de incidencia para el caso de Haití (1891 casos para 2008). Los otros países que llaman la atención por el número de casos creciente aunque con niveles bajos, son Brasil y Colombia (pasando de 34 a 38 casos para 2008). En el caso de

TABLA II. TENDENCIAS DEL GASTO EN SALUD EN RELACIÓN AL PIB DE CADA PAÍS SELECCIONADO

\begin{tabular}{lcc}
\hline País & 2002 & 2007 \\
\hline México & 6 & 7.5 \\
Brasil & 7.2 & 7.4 \\
Argentina & 8.9 & 7.9 \\
Haití & 4.2 & 5.5 \\
Colombia & 6.6 & 6 \\
El salvador & 5.8 & 6.9 \\
EUA & 12.3 & 12.3 \\
Canadá & 9.6 & 8.9 \\
\hline
\end{tabular}

Fuente: Elaboración propia a partir de Indicadores Básicos de Salud. Situación de Salud en las Américas. Info-OMS/OPS-2009. favorables en todos los países, excepto en Colombia, donde el gasto pasa de 6.6 a 6.0. No dejan de llamar la atención los casos de Canadá y EUA por los altos niveles de gasto en salud en relación al PIB.

\section{Tendencias y grado de avance en indicadores de} salud.

En lo referente a los niveles y tendencias para analizar los grados de avance en diferentes indicadores de salud, se resaltan en este documento los resultados para mortalidad infantil y sobre mortalidad materna. En el cuadro 3 se observan las tendencias de la mortalidad infantil para el período 1990-2008, así como los datos referentes a la tasa-meta acordada por cada país para el 2015 y el grado de avance alcanzado al 2008, último dato oficial reportado en el monitoreo de los objetivos del milenio, Informe ONU-2010. Es importante resaltar que de los 10 países que inicialmente se seleccionaron para el análisis, para el caso de este indicador solo fue posible tener datos confiables para Brasil, México, Argentina, Colombia, Canadá y EUA. Los casos que llaman la atención por los altos grados de avance alcanzado son Brasil y México, particularmente Brasil que al 2008 ya ha alcanzado la meta trazada para el 
2015. Los casos de Canadá y EUA presentan grados de avance mínimos por los bajos niveles ya alcanzados por estos países en las últimas décadas.

Los niveles, tendencias y grados de avance para el caso de la mortalidad infantil difieren de manera sustantiva al compararlos con los indicadores de mortalidad materna ( Tabla III). En efecto en mortalidad materna los grados de avance son menores. Aunque Brasil y México presentan los mayores grados de avance, ningún país alcanza los niveles de mejoría mas allá del $65 \%$, ni mucho menos haber alcanzado la meta programada para 2015. Por otra parte pasando a bajos niveles de avance, incluso de retrocesos o de tasa de avance negativa, no dejan de llamar la atención los casos de Colombia ( $-40 \%)$ y Canadá ( $-22 \%)$. En el caso de Colombia la tasa muestra tendencias crecientes de manera muy significativa en relación al resto de los países y seguramente esta asociado a incremento en los factores de riesgo de daños a la salud en etapa reproductiva y probablemente a problemas de subregistro que han ido mejorando con las últimas estadísticas. Para el caso de Canadá, la tendencia decreciente es minima y tiene que ver con la dificultad de mantener tasas tan bajas de mortalidad materna.

\section{DISCUSIÓN Y CONCLUSIONES}

Los resultados del análisis cualitativo y cuantitativo de la conformación de CMES, así como de las tendencias de indicadores de salud, gasto e inversión en salud, permiten plantear de manera explícita una serie de conclusiones para ser consideradas a manera de lecciones aprendidas que pudieran direccionar o redimensionar, según sea el caso, las CMES vigentes o por implementarse en los países de América Latina:

* La implementación de las CMES ha permitido incorporar el análisis de crisis del mercado en el contexto de la crisis del estado (sistema fragmentado, pertenencia de usuarios a economía formal o informal).

* En un contexto de reformas, las CMES reactivan el mandato de invertir en salud propuesto por agencias internacionales como OMS y Banco Mundial desde 1993 (22), o de mandatos acordados por presidentes de países de ONU durante la Cumbre de Monterrey, $2002(23)$.

TABLA III. TENDENCIAS Y ABATIMIENTO DE LA MORTALIDAD INFANTIL Y MORTALIDAD MATERNA A PARTIR DE METAS DEL MILENIO. (DISMINUIR LA MORTALIDAD INFANTIL EN DOS TERCERAS PARTES AL 2015 Y LA MORTALIDAD MATERNA EN TRES CUARTAS PARTES AL 2015)

\begin{tabular}{|c|c|c|c|c|c|c|c|c|c|c|}
\hline \multirow[b]{2}{*}{ País } & \multicolumn{5}{|c|}{ Mortalidad infantil } & \multicolumn{5}{|c|}{ Mortalidad materna } \\
\hline & 1990 & 2005 & 2008 & $\begin{array}{l}\text { Meta } \\
2015\end{array}$ & $\begin{array}{c}\text { Tasa } \\
\text { de } \\
\text { avance }\end{array}$ & 1990 & 2005 & 2008 & $\begin{array}{l}\text { Meta } \\
2015\end{array}$ & $\begin{array}{l}\text { Tasa de } \\
\text { avance }\end{array}$ \\
\hline Brasil & 56 & 22 & 18 & 20 & $105 \%$ & 220 & 110 & ND & 55 & $65 \%$ \\
\hline México & 45 & 20 & 17 & 15 & $93 \%$ & 110 & 60 & ND & 27.5 & $60 \%$ \\
\hline Colombia & 35 & 22 & 20 & 12 & $65 \%$ & 100 & 130 & ND & 25 & $-40 \%$ \\
\hline Argentina & 29 & 17 & 16 & 96 & $5 \%$ & 100 & 77 & ND & 25 & $31 \%$ \\
\hline Canadá & 8 & 66 & 3 & $40 \%$ & & 6 & 7 & ND & 1.5 & $-22 \%$ \\
\hline EUA & 11 & 8 & 83 & $37 \%$ & & 12 & 11 & ND & 3 & $11 \%$ \\
\hline
\end{tabular}

ND= Dato no disponible.

Fuente: Elaboración propia a partir de Metas del Milenio. Informe 2010, ONU. 
* La CMES, permiten sistematizar el monitoreo de los objetivos del Milenio-1990-2015, en materia de Salud y Seguridad Social, resaltando los los vínculos entre economía, salud y desarrollo.

* Las CMES contribuyen hacia un análisis integral del nivel y alcance de las metas en materia de mortalidad y acceso a los servicios de salud en grupos vulnerables; pero también al desarrollo de indicadores de gasto y costos esperados por tipo de daños a la salud o de grupo poblacional.

* La implementación de las CMES pueden generar un patrón de sistematización y funcionamiento irregular dependiendo del compromiso y vigencia política de los actores sociales que conforman dichas comisiones.

* El monitoreo de las propias CMES es altamente relevante ya que en algunos países las CMES permanecen como parte de una comisión coyuntural de principios de la década 2000-2010, más que un grupo de expertos ó masa crítica de avanzada que emita recomendaciones en materia de mayor alcance en metas de salud, economía y desarrollo a nivel de cada país.

* En la medida que más nos acerquemos al 2015, las CMES tendrán un rol de mayor pertinencia y relevancia, sobre todo en vísperas del análisis, monitoreo y alcance de los metas de los Objetivos del Milenio a sociados a macroeconomía y salud.

Finalmente, a manera de sugerencias se platnea desarrollar las siguientes acciones dependiendo del grado de avance de las CMES en cada país de la región: -Institucionalización de la CMES, mediante el desarrollo de su propio marco jurídico-legal, planes y reglas.

- Tomando como referencia las metas de los «Objetivos del Milenio» se propone ajustar metas más congruentes con el nivel de ingreso per cápita y el desarrollo socio-económico regional al interior de cada país .
- Seguir utilizando los indicadores y tendencias de financiamiento monitoreados por las CMES para sustentar mayores incrementos en la inversión pública en salud y plantear nuevos indicadores de asignación financiera.

- Es impostergable que cada Sistema Nacional de Salud proponga el desarrollo e implementación de mecanismos de transparencia y rendición de cuentas, con miras a mejorar la efectividad y eficacia del gasto en salud.

- De acuerdo a los indicadores de mortalidad infantil y materna monitoreados por las CMES, urge invertir más recursos en programas desarrollo infantil y prevención de mortalidad materno-infantil.

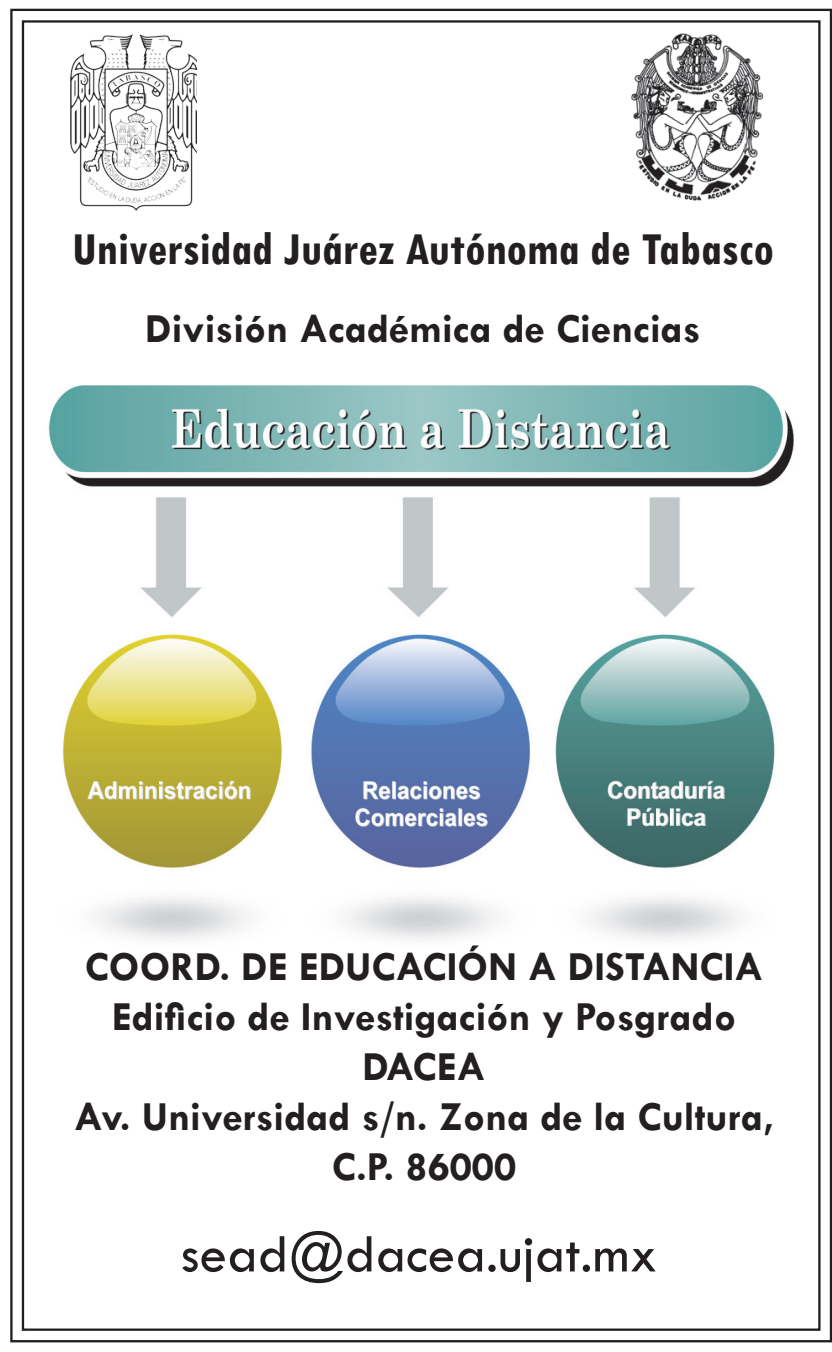


1. Secretaría de Salud. (2006). CMES-México. Macroeconomía y salud: Invertir en salud para el desarrollo económico. México: DF: Autor.

2. Galor, O. y J. Zeira. (1993). Income distribution and macroeconomics. Review of Economic Studies, 31(1), 35-53.

3. Mayer-Foulkes, D. (2001). The long-term impact of health on economic growth in Latin America. World development, 29(6), 1025-1033.

4. Ke, X. y Evans, D, Kawabata K. \& Riadh, Z. (2003). Household catastrophic health expenditure: a multicountry análisis. The Lancet, 362, 111-117.

5. Merlis, M. (2003). Family out-of-pocket spending for health services: a continuing source of financial insecurity. Working Paper. The Commonwealth Fund, 23-46.

6. Arredondo,A., Hernández, C., Orozco, E., y Álvarez, C. (2011). Estrategias de reforma y protección social en salud en México: segunda parte, efectos sobre equidad, eficiencia, cobertura y condiciones de salud. Cuad Med Soc de Chile, 50(4), 233-277.

7. Zúñiga, A. (2005). Health care costs and financial consequences of epidemiological changes in chronic diseases in Latin America: The Mexican case. Jou Public Health, 119(6), 711-720.

8. Hijar, M., Arredondo, A., Carrillo, C., y Solórzano, L. (2004). Road trafic in an urban area in México. An epidemiological and cost análisis. AccidentAnalysis and Prevention, 36(1), 37-42.

9. World Health Organization. (2005). Equity team, priorities for research to take forward the health equity policy agenda. Bulletin of the World Health Organization, 83(12), 948-953.

10. Programa de Reforma del Sector Salud. (20072012). Poder Ejecutivo Federal, México, D.F.

11. Nathan, P. (2005). Federalism and health policy. Health Affairs, 24(6), 1458-1465.

12. European Observatory of Health Systems. (2005). Health Care Systems in Transition, Organizational structure and management, 17-73.

13. Braveman, P., \& Gruskin, S. (2003). Poverty, equity, human rights and health. Bulletin of the World Health Organization, 81(7), 539-545.

14. Organización de Naciones Unidas. (2010). Los objetivos del milenio. Alcance de metas por país y región (Informe de la ONU) Recuperado de: milleniumindicators.un.org
15. Organización Mundial de la Salud. (2001-2006). Informes anuales de CMES-Ginebra. OMS.

16. World Health Organization. (2002). The CMH Report, 2002: its relevance for countries of the Latin America and Caribbean Region. Washington, DC: WHO/Pan American Health Organization. Versión en inglés disponible en: http://www.paho.org/English/DEC/ PAHOd.

17. Weil, N. (2005). Accounting for the Effect of Health on Economic Growth. NBER, Working Paper 11455.

18. World Health Organization. (2001). Commission on Macroeconomics and Health. Macroeconomics and health: investing in health for economic development. Geneva:WHO; Disponible en: http:// www3.who.int/ whosis/cmh/cmh_report/e/report. $\mathrm{cfm}$ ?path $=\mathrm{cmh}, \mathrm{cmh} \_$report\& language $=$english

19. Arredondo, A., y Orozco, E. (2009). Governance and equity from health care reform in Mexico. Inter. Jou of Health Planning and Management, 23, 37-49

20. Arredondo, A., y Nájera, P. (2008). Equity and accessibility in health? out-of-pocket expenditures for health care in middle income countries. Jou Pub Health/Cad de Sau Pub. Brasil, 24(12), 2819-2826.

21. Organización Panamericana de la Salud, (2010). Situación de Salud en las Américas, Edición 2010. Washington DC: OPS.

22. Fernández, J., Pereira, J., y Torres, A. (1995). Una agenda a debate: el informe del Banco Mundial «invertir en salud». Rev. Esp. de Salud Pública, 69, 385-391.

23. Organización de Naciones Unidas. (2002). Conferencia Internacional sobre Financiación para el desarrollo. Monterrey, México. Res ONU-59/293. 